\title{
Regard ethnographique sur les fonctions sociales de la pirogue à pagaie chez les Bushinengé de
} Guyane

An Ethnographic View of the Social Functions of Paddled Pirogue in the Tribe of Bushinans in French Guiana

Jérôme Pruneau, Jacques Dumont, Nathalie Agoudouman et Frédéric Bourdon

\section{(2) OpenEdition Journals}

Édition électronique

URL : http://journals.openedition.org/rref/794

DOI : $10.4000 /$ rref.794

ISSN : 2494-7830

Éditeur

CRDP de Guadeloupe

Édition imprimée

Date de publication : 15 décembre 2009

Pagination : 43-52

ISSN : 1962-2864

Référence électronique

Jérôme Pruneau, Jacques Dumont, Nathalie Agoudouman et Frédéric Bourdon, « Regard

ethnographique sur les fonctions sociales de la pirogue à pagaie chez les Bushinengé de Guyane »,

Recherches et ressources en éducation et formation [En ligne], 3 | 2009, mis en ligne le 24 avril 2020, consulté le 15 octobre 2020. URL : http://journals.openedition.org/rref/794 ; DOI : https://doi.org/ 10.4000/rref.794

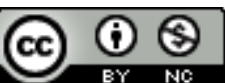

La revue Recherches et ressources en éducation et formation est mise à disposition selon les termes de la Licence Creative Commons Attribution - Pas d'Utilisation Commerciale. 


\title{
REGARD ETHNOGRAPHIQUE SUR LES FONCTIONS \\ SOCIALES DE LA PIROGUE A PAGAIE CHEZ LES BUSHINENGÉ DE GUYANE
}

\section{J. PRUNEAU (1), J. DUMONT (1), N. AGOUDOUMAN (1) ET F. BOURDON (1)}

(1) Laboratoire ACTES, UPRES EA 3596, Université Antilles-Guyane, Campus de Fouillole, BP 250, 97157 Pointe-a-Pitre Cedex

\section{Contact : pruneau.j@wanadoo.fr}

Résumé : Par le biais d'une enquête ethnographique auprès des Djuka, des Saramaka et des Aluku de Guyane française, il s'agit d'appréhender les fonctions sociales de la pirogue à pagaie chez les Bushinengé de Guyane. Cette embarcation jouait un rôle important dans les fonctions sociales des groupes : transport des biens et des personnes, pêche, chasse et cueillette, contact familial, rites d'apprentissage des techniques pour les plus jeunes ou encore jeux pour les enfants ou pour les adultes (courses). Pourtant, au fil des époques, la pirogue va subir des mutations à vocation économique jusqu'au milieu du $20^{\mathrm{e}}$ siècle (transport du bois - milieu 19e ; orpaillage fin $19^{\mathrm{e}}$ ), où l'utilisation, les techniques de navigation et les matériaux de la pirogue se modifient fortement avec l'apparition du moteur hors-bord, dont la multiplication d'utilisation s'accroît après les années 1950-1960 au point de faire quasiment disparaître la forme traditionnelle de pratique.

Mots clés : Ethnologie, pirogues à pagaie, fonctions sociales, Guyane française, tradition.

\begin{abstract}
Through an ethnographical inquiry in the Djuka, Saramaka and Aluku tribes of French Guyana, this study is about understanding the social meanings and functions of the practice of pirogue with paddles in the Bushinengés of French Guyana. This small crafts used to play an important role in the group social functions: carriage of goods, transportation of people, fishing, hunting and gathering, family linkage, initiation rite for the learning of techniques for the youngest or games for children and adults (races). Yet, throughout the times, the pirogue has been transformed through economic-based mutations until the mid- $20^{\text {th }}$ century (wood carriage mid- $19^{\text {th }}$ century; gold washing - late $19^{\text {th }}$ century), when the use, the sailing techniques and the materials of the pirogues have been strongly modified with the introduction of the outboard motor, whose use has increased after the 1950 s-' 60 s to the extent that the traditional practice has nearly disappeared.
\end{abstract}

Keywords : Ethnology, pirogues with paddles, social functions, French Guyana, tradition. 


\section{INTRODUCTION}

Les embarcations ont toujours été présentes dans les civilisations vivant au bord de l'eau (rivières, fleuves, mers), qu'il s'agisse de pirogues amérindiennes, africaines, polynésiennes, asiatiques, océaniques ou encore caribéennes. En fonction de la géographie, du relief, de l'économie ou de l'histoire, chaque culture a fabriqué des embarcations adaptées à son environnement et à ses besoins. Les populations d'origine africaine en Guyane française, les Noirs marrons ${ }^{1}$ appelés également " Bushinengué $»^{2}$, ont eu recours à la pirogue à pagaie (palo boto) dès la fin du $18^{\text {ème }}$ pour franchir les fleuves du Surinam et de la Guyane afin de fuir la colonisation. L'utilisation de ce type d'embarcation n'est pas nouvelle pour ces populations puisque déjà pour l'agriculture coloniale au Surinam, les voies de communication étaient liées au système de « polder » qui obligeait à creuser des canaux. Les maitres étaient transportés par les esclaves dans des pirogues à pagaie d'une plantation à l'autre et les marchandises étaient acheminées vers le port. Plus tard, à l'indépendance de ces ethnies, les fonctions sociales du groupe étaient directement liées à l'usage des pirogues : transport des biens et des personnes, pêche, chasse et cueillette, contact familial, rites d'apprentissage des techniques pour les plus jeunes ou encore jeux pour les enfants ou pour les adultes (courses). Cependant, au fil du temps, la pirogue va subir des mutations à vocation économique jusqu'au milieu du $20^{\text {ème }}$ siècle (transport du bois - milieu 19 $9^{\text {ème }}$; orpaillage - fin 19 $9^{\text {ème }}$ ), où l'utilisation, les techniques de navigation et les matériaux de la pirogue se modifient fortement avec l'apparition du moteur hors-bord, dont la multiplication d'utilisation s'accroît après les années 1950-1960 au point de faire quasiment disparaître la forme traditionnelle de pratique. Face à cela, les populations semblent aujourd'hui mettre en place des dispositifs visant à redéfinir l'utilisation originelle de ces pirogues à des fins de préservation, en réorientant par exemple la fonction sociale et économique de la pirogue en objet culturel et de patrimoine. Mais ce processus n'en est qu'à ses balbutiements contrairement à d'autres activités traditionnelles dont la trajectoire a suivi ce type de mécanisme permettant la revitalisation des pratiques (Guttman, 1979 ; Callède, 1990 ; Camy, 1995 ; Bromberger, 1995 ; Le Goff, 1996 ; Pfister, 1999 ; Pégard et Pruneau, 2001 ; Pruneau, 2003 ; Pruneau, Dumont et Célimène, 2006). Par le biais d'une enquête minutieuse auprès des Djuka, des Saramaka et des Aluku dont il existe peu de traces écrites (Price, 2003), il ne s'agit pas ici de s'intéresser à ces processus mais davantage de comprendre quelles étaient les fonctions de cette embarcation au sein de ces groupes culturels.

\section{LA PIROGUE À PAGAIE CHEZ LES BUSHINENGUÉ}

C'est du Hollandais Boschnegers ou Bosnegers et de l'anglais Bush Negroes que provient l'étymologie du mot Bushinengué. On les appelle aussi Maawina Nengué (les Nègres du Maroni) ou encore Liba Piking (les enfants du fleuve). Ils sont d'« anciens esclaves marrons qui ont fui le Surinam au XVIII siècle et sont parvenus à constituer au sein de la forêt des entités politiques relativement autonomes. Ils ont en grande partie préservé des usages et un mode de vie hérité du continent noir » (Delisle, 2000 : 11). Ils sont Djuka, Saramaka, Matawaï, Kuinty, Paramaka et enfin Boni ou Aluku3 ${ }^{3}$, soit six ethnies constituées en sociétés matrilinéaires. Ces groupes se différencient entre eux à trois niveaux. Un premier niveau, linguistique, démarque ces ethnies par des structures langagières qui, si elles permettent pour certains groupes de se comprendre (Djuka, Paramaka et Aluku d'un côté, Saramaka, Matawaï et Kuinty de l'autre), n'ont pas les mêmes origines : anglaises pour les premiers, portugaises pour les seconds. Un deuxième niveau, géographique, répartit ces ethnies selon des territoires définis : les Saramaka, les Matawaï et les Kuinty sont installés principalement au Surinam alors que les Djuka, les Paramaka et les Aluku vivent essentiellement le long des fleuves Maroni et Lawa ${ }^{4}$ en Guyane française. Enfin, un troisième niveau, historique, marque l'indépendance institutionnelle des uns et des autres dans un temps chronologique distinct. L'histoire des Aluku est difficile à constituer, notamment en ce qui concerne la date de leur formation. Tristan Bellardie pensent qu' 'ils se sont formés vers 1772, autour d'un chef, Boni » (Bellardie, 1994 : 27) alors que Jean Moomou estime que "d'après Wim Hoogbergen, les archives notent leur existence réelle en 1769, mais le noyau initial remonte à 1712 comme les autres groupes » (Moomou, $2004: 105$ ). Si vraisemblablement leur installation en Guyane française date de la fin du $18^{\text {ème }}$ siècle, leur indépendance est déclarée sous présence française à la conférence d'Albina le 8 septembre 1860. À la différence de tous les habitants des vieilles colonies, les Aluku n'ont pas le statut de citoyen et demeurent des sujets français jusqu'en 1969 où ils acquièrent la citoyenneté en même temps que la création de communes sur le Maroni. A l'inverse, l'histoire des autres ethnies est plus facilement repérable, les Djuka, les Saramaka, les Matawaï, les Kuinty et les Paramaka apparaissant en amont des fleuves du Surinam suite aux événements de $1712^{5}$. Ils parviennent à conclure des traités de paix avec les Hollandais dans la deuxième moitié du $18^{\text {ème }}$ siècle.

Chez les Bushinengué, trois types de pirogues à pagaie peuvent se distinguer. En effet, les pirogues des Djuka et des Saramaka qui, elles-mêmes, ne sont pas identiques entre elles, se différencient par leur forme de celles des Aluku. Pour autant, la pirogue des Aluku se rapproche plus de celle des Djuka que celle des Saramaka. D'après Madeleine Makélé, veuve d'un capitaine de Cottica et petite-fille du Gran Man Ochi ${ }^{6}$ (cf. Infra) et Antoine Bayonne, fils d'un capitaine de Cottica, les Saramaka fabriquent des pirogues à pagaie très larges au milieu avec une toute petite pointe avant et arrière, voire une inexistence de pointe arrière. Ainsi constituées, les embarcations 


\section{J. PRUNEAU, J. DUMONT, N. AGOUDOUMAN ET F. BOURDON}

sont plus ventrues, donc moins rapides, mais favorisent à l'inverse le transport de marchandise ou de matériel. Par contre, "Les Djuka ont une forme de canot plus évoluée, plus effilée à l'avant et plus relevée à l'arrière ; les Boni ont poussé à l'extrême la recherche de la pureté de la ligne; leurs canots se reconnaissent de loin à leur pointe avant presque rectiligne et très basse sur l'eau, alors que l'avant des canots Djuka est légèrement incurvé » (Ibid.).

Les Djuka et les Aluku vivant aux abords de fleuves jalonnés de sauts, ce relief de leur lieu d'habitation influence la construction des pirogues afin de pouvoir franchir ces obstacles naturels sans trop de difficulté, contrairement aux Saramaka qui construisent des pirogues davantage adaptées à la navigation en mer. Au final, ce qui fait la spécificité des pirogues bushinengué repose sur trois points qui ne seront pas pour autant détaillés plus avant ici : 1) les bordés en planche permettent un chargement plus important de la pirogue tout en évitant de prendre de l'eau lors des passages des rapides ; 2) les pointes aux extrémités qui dépassent au-dessus du bordage sont un signe de reconnaissance, dédoublé d'une volonté d'appartenance à travers les dessins, les timbé, représentatifs notamment chez les Djuka et les Aluku ; 3) l'ouverture au feu de la coque monoxyle. Au-delà de la différence dans les techniques de construction et de l'objet en tant que tel, l'usage de cette embarcation dans ces communautés est en lien direct avec leur vie quotidienne et la pirogue à pagaie concentre un certain nombre de fonctions sociales qu'il convient d'éclairer afin de comprendre toute leur utilité.

\section{LA PIROGUE À PAGAIE ET SES FONCTIONS SOCIALES}

La pirogue à pagaie, au-delà de l'objet pratique, sert de multiples fonctions qui dépassent sa fonction première et participent à la constitution du groupe, à sa cohésion, à sa structuration sociale et familiale. En cela, elle tient lieu d'élément fondamental de l'identité des Bushinengué. La première fonction de cette embarcation remonte au marronnage, ces peuples et en particulier les Aluku, ayant utilisé cet engin pour échapper aux colonisateurs. Comme tous les autres groupes, leur fuite dans la forêt les a menés jusqu'aux abords des fleuves du Surinam et de la Guyane. Là, il semblerait que les Aluku aient d'abord utilisé les canots des Amérindiens Galibi pour s'installer sur la rivière Sparouine en 1776 et fuir le monde colonial : "Les Boni sont allés chez les Galibi (tribu amérindienne) de l'estuaire [...], ont pris avec violence leurs embarcations, [...] et franchissent le Maroni » (Moomou, op. cit. :119). Mais pour progresser vers le haut Maroni, ces populations ont recours à la construction des pirogues, sésames pour le transport de l'ensemble des individus qui composaient ce groupe. C'est ainsi, qu'étapes par étapes, ils s'installent à « Bonidoro », " Gaan Daaï », puis aux environs de "Lessé Dédé », «Cottica », « Gaan Maawina » pour redescendre par la suite.

Si la pirogue à pagaie a joué un rôle fondamental dans l'évasion des Aluku, elle participe également à «la formation d'un isolat humain " (Ibid. :135), une société géographiquement loin du monde colonial et quasi autonome. En effet, au lendemain de leur indépendance, les Aluku mettent en place des règles de fonctionnement véritablement dissociées des institutions colonialistes, mais beaucoup plus proches de l'organisation sociale de certaines tribus africaines. La société Aluku s'organise selon une structure sociale déterminée de type totémique (faakatiki), en lien étroit avec celles des autres Bushinengué : le Gran Man, chef suprême des Aluku, est « le détenteur de l'exécutif », le Fishcali et le Mayoo « les officiers de justice supérieure », le Capitaine et le Bassia « les officiers de justice inférieure ». Chacun tient ici un rôle précis, forme de conseiller particulier : le Fishcali est à la fois l'administrateur du foncier, connaissant les parcelles de terres accordées à chaque membre de la population, à la fois le porte parole du Gran Man. Le Mayoo est le conseiller privilégié du Gran Man. Le Bassia est responsable d'une partie géographique du village, alors que le Capitaine, lui, est le représentant d'un village, un édéman fu condé, sorte de sous-chef. Cette structuration sociale distincte du modèle occidental a permis à cette ethnie Bushinengué de maintenir son propre univers culturel et social. Mais cette autonomie des Aluku n'a été que relative par le fait d'un contact régulier avec le monde colonial et relativement continu, particulièrement à partir de « la ruée vers l'or » (cf. Infra), et notamment par la nécessité de s'approvisionner en matériel : couteau, sabre, fusil, poudre, etc. Dans ce schéma de relation économique, la pirogue à pagaie a servi de moyen de transbordement dans l'approvisionnement de ces produits achetés par les Aluku à Saint-Laurent en descendant du Haut Maroni.

L'organisation sociale des groupes et leur répartition géographique n'ont guère changé de nos jours, ces populations étant installées de part et d'autre du fleuve Maroni ${ }^{7}$ long de 520 kilomètres, frontière naturelle entre le Surinam et la Guyane Française. Depuis les années $1990^{8}$, une piste dessert cette région de la Guyane joignant ainsi la commune de Maripasoula à celle de Papaïchton. Pour autant, malgré la liaison aérienne quotidienne entre Maripasoula et Cayenne, la pirogue reste le moyen de transport incontournable pour les voyageurs n'habitant pas au bourg de cette commune, la route reliant la commune d'Apatou à Saint-Laurent du Maroni étant ellemême actuellement en construction. La voie fluviale reste donc le moyen privilégié de communication pour ces populations : "Les autoroutes chez les communautés Bushinengué c'est le fleuve. [...] C'est le Maroni "9. Par conséquent, la pirogue se présente comme l'ultime et unique moyen de transport des biens et des personnes, nécessitant pourtant des trajets plus ou moins longs en bateau. Au-delà de l'aspect de communication, la pirogue 
s'inscrit aussi dans le quotidien des Bushinengué, comme engin de déplacement, d'aide à l'agriculture, à la chasse, à la pêche. En effet, l'usage de cette embarcation permet d'accéder à tous les produits de première nécessité qui se trouvent rarement dans le village, produits issus d'une agriculture itinérante sur brûlis, de la pêche, de la chasse et de la cueillette.

Le seul exemple de l'agriculture suffit à en comprendre les tenants et les aboutissements. Celle-ci est réalisée dans des abattis coupés, déboisés, brûlés, nettoyés par les hommes et situés en amont ou en aval du fleuve, parfois sur la rive opposée au village, souvent d'ailleurs du côté surinamien. Les femmes et les enfants (quelques fois leur mari) y cultivent du manioc pour la fabrication du couac ${ }^{10}$, aliment de base des Bushinengué, mais aussi du maïs, des cacahouètes et de la canne à sucre, du riz, des tubercules, des légumes ou encore des fruits (bananes, ananas) à des fins de consommation propre. Utilisée dans ce cas par les femmes, la pirogue permet, après la récolte, le transport des produits issus de l'abattis, tout comme elle permet d'aller dans les endroits destinés au ramassage des graines de maripa indiquées pour la fabrication de l'huile de consommation courante ou de l'huile à des fins esthétiques pour les cheveux ou le corps. De même, la récolte des graines d'awara leur est dévolue, afin d'en extraire une huile (tchotcho) qui sert pour le massage ou à des fins thérapeutiques, notamment lorsque quelqu'un se blesse. Dans ce cas, le Dééchiman ${ }^{l 1}$ installe le blessé dans une maison à part (tapu na ossu) où l'accès est limité surtout pour les femmes indisposées (symbolique de l'impureté du sang), afin de lui promulguer des massages. Le blessé ne doit pas avoir de relations sexuelles durant ce laps de temps de convalescence, ni même parler aux passants. Ces précautions sont prises afin d'éviter une «non-efficacité » de la thérapie (poli a dééchi). Mais le tchotcho a d'autres usages, comme celui lié à la préparation des morts. Lorsque le corps du défunt tend à se raidir, l'huile tchotcho facilite un relâchement des articulations, nécessaire à la toilette du corps. Lors de la séance mortuaire, seule une femme ménopausée peut préparer cette huile, la femme en âge de procréation appartenant là encore au domaine de l'impure, source d'une possible inefficacité voire d'une inhibition de l'effet recherché. L'emploi de la pirogue par les hommes fait référence aux tâches mécaniques (Durkheim, 1912) qui leur sont dévolues. Durant plusieurs jours, voire pendant une semaine, il arrive que les hommes partent pêcher (à la ligne et/ou au filet), cueillir ou chasser pour ramener du pingo (cochon bois) ou du kumalu (coumarou), mets particulièrement appréciés de leurs épouses. La pêche peut se pratiquer soit en dérivant dans la pirogue (dongo $u k u$ ), soit dans une pirogue restée accrochée sur un rocher (baka chiton). Mais, dans ces deux cas, la pirogue devient un outil technique à part entière, tout comme lors de la chasse qui, si elle se fait généralement dans la forêt, est parfois pratiquée sur l'eau dans l'attente des gibiers qui traversent le fleuve. Dans le cas de la cueillette, les hommes, pour faire plaisir à leur(s) femme $(\mathrm{s})^{12}$ qui raffolent du jus obtenu par les graines de palmiers $($ comu, patawa, apodo (wassaï), utilisent la pirogue pour remonter le fleuve à la recherche de ces graines, tout comme ils profitent de ces temps pour ramener des feuilles de palmiers ${ }^{13}$ indispensables à la construction des habitations.

La pirogue sert une autre fonction qui participe à l'ordre familial. En général, les villages sont habités par des lo - Clan, lignage - (par exemple, Boniville est habité par le lo Dikan, Loca par le lo Lapé, Assissi par les lo Dju et Dipélu, l'Enfant Perdu et Apatou par le lo Dipélu, etc.). Mais il peut cependant y avoir des membres d'une même famille dispersés dans d'autres campu (campements) ou dans différents villages environnant. La pirogue devient alors le lien permettant le contact entre eux, notamment lorsque les parents confient à leurs enfants une course à faire chez un membre de la famille habitant un autre village. D'ailleurs, dès leur plus jeune âge, les enfants empruntent pour l'occasion des pirogues à des gens du village ou se baladent dans les pirogues de leurs parents. Tel un rite initiatique, l'apprentissage pour manœuvrer une pirogue à pagaie s'effectue sur les conseils des adultes lors des séances de pêche ou lors des voyages à l'abattis par transmission orale. C'est ainsi que dès l'âge de 8-9 ans, les enfants barrent seuls une pirogue à pagaie qui, bien souvent, devient aussi un excellent instrument de jeu.

Enfin, la pirogue à pagaie remplit une fonction sociale lors des cérémonies funéraires. Les cimetières Bushinengué ne se trouvant pas dans les villages, les villageois utilisent les pirogues pour mener les morts à leur dernière demeure. Puis elle sert pour ramener les effets du mort (wiwï : ongle et cheveux), essentiels pour le « questionner» sur les raisons de son décès, après le bookodél ${ }^{14}$. Un an plus tard, les villageois organisent une pêche particulière au nivrée $(p o n s u)^{15}$ pour préparer les mets servis lors de la levée de deuil ${ }^{16}$, cérémonie (doo udu) durant laquelle la population revêtue de tenues traditionnelles (pagnes brodés) monte dans des pirogues pour réaliser des ballets nautiques au milieu du fleuve. Dans son rôle traditionnel, la pirogue marque ici l'identité culturelle et sociale des Bushinengé, et notamment des Aluku. Si ces fonctions sociales organisent et participent du quotidien, une autre fonction de la pirogue va engendrer sa transformation et son évolution, notamment dans sa technique d'utilisation : la fonction économique.

\section{LA MUTATION DE LA PIROGUE À PAGAIE PAR SA FONCTION ÉCONOMIQUE}

Nombreux sont les Aluku et Djuka qui investissent le secteur du bois à partir de la loi sur le bois votée au Surinam en 1856, "qui facilite la participation des Marrons à cette activité économique » (Price, op. cit. : 43). Par la réalisation « des trains de flottage », ils acheminent le bois jusqu'aux dépôts hollandais et/ou français. Alors trop 


\section{J. PRUNEAU, J. DUMONT, N. AGOUDOUMAN ET F. BOURDON}

petites pour contenir le bois, les pirogues à pagaie deviennent essentielles pour encadrer les « radeaux » flottants qui descendent jusqu'au point de vente du matériau précieux. Dans ces lieux de commerce, les Bushinengué s'approvisionnement avec leur paye avant de remonter dans leur village lors de voyages qui, à contre-courant, pouvaient durer des semaines, voire des mois selon la saison (des pluies ou sèche). Pour autant, malgré ces incursions en milieu occidental, "les contacts des Aluku avec la société de la côte ont [...] toujours été, à cette époque, assez limités » (Ibid. :44). C'est une autre activité bien plus prolifique que celle du bois qui va prendre forme à la fin du $19^{\text {ème }}$ et au début du $20^{\text {ème }}$ siècle et qui va multiplier les contacts : celle de l'orpaillage.

La découverte de l'or sur le haut-Maroni dès 1883 sur la Tampok, 1887 sur le Lawa et 1900 sur l'Inini ${ }^{17}$, va en effet propulser les bushinengué et leurs pirogues sur le devant de la scène. Dès "1877, [on] note l'existence en pays Aluku d'un placer ${ }^{18}$ occupant un millier d'ouvrier $»^{19}$. D'année en année, ce nombre augmente jusqu'à cinq à six mille personnes, occasionnant des répercussions directes sur la densité de circulation entre les villes de Saint-Laurent et Albina et les villages des orpailleurs : les hommes, le matériel, la nourriture sont autant de convoyages à mettre en place entre les villages de travailleurs et la côte, et vice-versa. Cet affairement oblige non seulement la présence d'une multitude de pirogues, mais aussi la nécessité d'un savoir-faire d'experts pour qui la rivière, difficile à naviguer, n'a pas de mystère. Incontournables, puis irremplaçables, les Bushinengé sont pour R. Maufrais « les rois des rivières de Guyane » (Maufrais, 1949 : 358), alors que pour S. Mam Lam Fouck: " la conduite des embarcations réclamait des hommes compétents afin de limiter les risques de naufrage... Ces compétences devaient être recherchées parmi les Bonis et les Saramacas... » (Mam Lam Fouck, 1999 : 244). Très vite, les Bushinengué comprennent le jeu de dépendance avec les orpailleurs, tout comme ils intègrent l'enjeu de la manne financière qui l'accompagne. Au point où, de manière indirecte, la valeur de l'or elle-même s'aligne sur leur activité (augmentation des prix de convoyage, fixation arbitraire du prix, etc). Cette inflation entraîne d'ailleurs de vives réactions de la part des orpailleurs auprès du gouverneur de la Guyane, arguant le non-respect des accords passés en 1878 avec le Gran Man des Aluku, Anato, accords qui stipulaient que le coût du transport était de trois francs au maximum (Benoit, 2000). De leur côté, les Alukus rétorquent en invoquant que ces augmentations sont liées à la dangerosité associée aux navigations : " pour franchir un rapide ou une chute, il faut que les hommes pagayent de toute leur force, car on ne peut diriger une embarcation d'autant que sa vitesse est plus grande que celle du courent »(Crevaux, 1987 : 55).

La clé de ces débats s'appuie en fait sur la saisonnalité, la navigation sur le fleuve étant marquée par deux grandes périodes : la saison sèche d'août à novembre (saison des basses eaux) et la saison des pluies de décembre à juillet (saison des grandes eaux). À la saison sèche, les piroguiers ne peuvent éviter le " mama mofu », autrement dit la voie principale du fleuve. Pendant cette période, la navigation est d'une extrême pénibilité, ce qui la rend particulièrement dangereuse. Le déchargement d'une bonne partie de la cargaison est fréquent du fait du franchissement de la plupart des sauts. Si ces précautions diminuent les risques de naufrage (la pirogue est allégée pour ceux qui la tirent pour remonter le saut), l'ensemble du chargement ainsi que les passagers doivent être transportés par les piroguiers de l'aval du saut à son amont en empruntant la voie terrestre. Ces manœuvres sont coûteuses en temps et en énergie, et si les Alukus de l'époque élevaient leurs tarifs de transbordement, aujourd'hui encore, les prix du fret en pirogue sont majorés durant cette saison. Pendant la saison des pluies, la navigation est plus facile, la remontée ou la descente du Maroni est moins périlleuse. Les piroguiers empruntent essentiellement les bistouris " buchi ini wata pachi », réseau de voies navigables parallèles qui permet de contourner la plupart des grands sauts. Toutefois, ces passages font appel à une excellente connaissance du milieu sous peine de s'égarer dans les multiples méandres des îles qui jalonnent le fleuve Maroni. Encore de nos jours, rares sont ceux qui connaissent parfaitement ces voies. D'après le Fishcali Louis Topo ${ }^{20}$ le voyage pouvait durer jusqu'à une semaine à cette période. Mais à mesure que le niveau de l'eau baisse, ces bistouris s'assèchent et deviennent de moins en moins praticables.

Par ailleurs, le coût du voyage était également déterminé en fonction du sens qu'il empruntait : remonter ou descendre le fleuve est une aventure bien différente. Si à la fin du $19^{\text {ème }}$ début du $20^{\text {èue }}$ siècle, les pirogues descendaient et/ou remontaient avec une cargaison qui était plus importante au moment de la descente ${ }^{21}$, dans la deuxième moitié du $20^{\text {ème }}$ siècle la tendance s'est inversée : les pirogues sont dorénavant plus chargées lors de la remontée par le fait d'une multiplication des ménages installés sur le haut du fleuve qu'il faut ravitailler. Pour autant, ces remontées bénéficient aujourd'hui de l'apparition du moteur. S'il est vrai qu'une pirogue vide est plus facile dans les manœuvres à descendre qu'une pirogue chargée, lors de la remontée à contre-courant, l'exercice se corse davantage. Sollicités deux fois plus, en particulier dans les rapides par la résistance à l'avancement de la pirogue, les pagayeurs souffrent beaucoup et se fatiguent plus vite. D'où une possible explication dans les cas de naufrages répertoriés chez les Bushinengué dans le sens de la remontée (encore aujourd'hui).

Toujours est-il que cet âge d'or des Bushinengé lié à « la ruée vers l'or » marque la période où ils ont été, en particulier les Aluku, en contact prolongé avec « le monde extérieur ». Ce brassage continu a permis leur enrichissement financier (et culturel ?), au point où les villages Bushinengué se sont vite retrouvés submergés de produits du monde colonial, signe d'un temps nouveau, notamment dans les premières années du $20^{\text {ème }}$ siècle : 
consommation de nouvelles marchandises, apparition et apprentissage de nouveaux savoir-faire, apparition et appropriation de nouvelles technologies sont autant d'éléments qui participent d'une acculturation. C'est ainsi qu'on note l'apparition du moteur hors-bord dans les rapports des missions réalisées dans les années 1930 : «L'un de nos canots, équipé avec un propulseur à essence nous permis de constater les nombreux avantages de ce mode de locomotion, malgré les rapides et les sauts » (Goreaud, $1931: 3)$. Le contrôleur forestier Vaillant ajoute : "La tournée a été effectuée avec le canot Boni, 2 canotiers et le moteur 9 CV Johnson » (Vaillant, $1937: 14$ ), quand le Docteur Douvier affirme : «Les moyens de transport ont été les suivants : au départ de la forestière le grand canot Tumuc Humac et le moteur Johnson 9 CV [...] après l'accident survenu au moteur 22 CV 》 (Douvier, 1938 : 43). Le nombre des moteurs est très réduit à cette époque et ceux utilisés lors de ces missions appartenaient à l'administration chargée de faire des tournées de contrôle dans le territoire de l'Inini. Le développement de la navigation au moteur sur le Maroni ne se réalise véritablement que dans les années 1950, leur appropriation par les Bushinengé encore plus tardivement, à en croire Antoine Bayonne ${ }^{22}:$ le premier Aluku à posséder un moteur est Monsieur Galimo (futur revendeur) de Papaïchton dans les années 1960.

Bien sûr, l'apparition de cette technologie n'est pas sans conséquences sur l'usage et l'objet même de la pirogue. Des modifications sont apportées dans la construction pour permettre d'embarquer le moteur, tout comme l'avènement de nouveaux matériaux comme le plastique modifie certains aspects de la pirogue. En effet, les pirogues à pagaie construites à partir des années 1880 arboraient toutes un petit abri dénommé « boto ossu » ou « pamakaï ». Ce « boto ossu » assurait l'étanchéité durant les parcours et facilitait le rangement des effets personnels. Il abritait les missionnaires durant le trajet, laissant place la nuit aux piroguiers qui y dormaient dessous et surveillaient l'embarcation et sa cargaison. Le « boto ossu » mesurait environ 2 mètres de long, entièrement conçu en paille. Si l'apparition de la bâche dans les années 1920 a peu à peu diminué sa fabrication jusqu'à sa quasi-disparition, son utilisation resurgissait parfois à des fins de prestige et d'authenticité lors des déplacements du sous-préfet et des hommes de l'administration, voire sur quelques taxis pirogues qui effectuaient la navette entre Saint-Laurent du Maroni et Albina.

La deuxième moitié du $20^{\text {ème }}$ siècle et son corollaire d'évolutions sociale, économique, technique et technologique, ont participé à la transformation de la pirogue à pagaie, puis à sa perdition progressive dans les années 1990, au point de faire de cette embarcation un objet dont l'utilité sociale s'est égrenée au fil du temps. Dans sa forme traditionnelle, la pirogue à pagaie est aujourd'hui très menacée, chez les Aluku notamment (seules une ou deux pirogues subsistent mais n'ont pas été construites par eux). Si face à ce phénomène en déclin quelques initiatives semblent émerger pour redonner vie à cet objet social et culturel si longtemps ancré dans les traditions de ces sociétés, elles ne le sont malheureusement pas par les usagers Bushinengé eux-mêmes. Le processus qui participe de cette volonté de (re)dynamiser la pirogue s'inscrit dans la mise en place, par des personnes extérieures à ces groupes culturels, d'événements ludiques et sportifs. Reste à espérer que ceux-ci permettent la réutilisation de cette embarcation, certes à des fins différentes, mais de manière suffisamment efficace pour voir se conserver un objet dont la vocation sociale d'hier pourrait se transformer aujourd'hui en vocation patrimoniale.

\section{CONCLUSION}

La pirogue à pagaie des Bushinengé a constitué un objet central dans la vie sociale de ces sociétés jusqu'à récemment, voire dans leur survie en s'inscrivant dans des fonctions précises pour l'ensemble du groupe. La transformation économique et sociale fondamentale du $20^{\text {ème }}$ siècle marque un coup d'arrêt dans l'usage traditionnel des pirogues au point de mettre en péril leur destinée. Si le processus de «sportivisation » réussit à revitaliser certaines pratiques traditionnelles (Pruneau, Dumont, Célimène, 2006) aux Antilles, les quelques initiatives engagées dans les pirogues à pagaie en Guyane sont contingentes à une participation plus massive des pratiquants pour en avoir les effets escomptés, même si quelques compétitions locales et nationales entre 1997 et 2006 ont permis une relative relance de la construction des embarcations. Par contre, la prise de conscience des acteurs peut permettre une mise en patrimoine progressive de la pirogue en tant qu'objet culturel ou « objet symbole » selon l'expression de Chastel (1994). À ce titre, une démarche symbolique du musée des cultures guyanaises de Cayenne signe la volonté de cette (re)naissance par une commande exclusive adressée au capitaine Saramaka Adaïsso d'une pirogue et de différentes pagaies pour les exposer. Ces actions locales s'inscrivent peut-être comme la clé d'une réappropriation possible d'un pan de cette culture séculaire, en lien étroit avec une intégration récente de ces populations de l'intérieur qui, avant les années 1990, apparaissaient encore en marge d'une société créole guyanaise largement implantée sur le littoral de ce département français d'Amérique.

\section{BIBLIOGRAPHIE}

- Bellardie, T. (1994). Les relations entre Français et Boni en Guyane française. Processus de colonisation et dépendance à travers le problème frontalier du Maroni 1836-1893. Mémoire de Maîtrise de l'université de Toulouse, septembre, $148 \mathrm{p}$. 
- Bilby, K. (1987). Les Boni et les communes : un problème d'intégration. Equinoxe, 24, 100-111.

- Bromberger, C. (1995). Le match de Football. Ethnologie d'une passion partisane à Marseille, Naples et Turin. Paris : M.S.H.

- Callède, J. P. (1990). La pelote basque. Expression culturelle et/ou pratique sportive. E.P.S., 224, 75-78.

- Camy, J. (1995). Les quilles en Gascogne. Terrain, 25, 61-72

- Chastel, A. (1994). Architecture et patrimoine. Paris : Imprimerie nationale.

- Crevaux, J. (1987). Le Mendiant de l'Eldorado. De Cayenne aux Andes 1876-1879. Paris : éditions Phébus.

- Delisle, P. (2000). Le Rêve d'une Guyane «toute chrétienne », les pères du Saint Esprit sur le Maroni et sur l'Oyapock dans la seconde moitié du $19^{\text {ème }}$ siècle ». In Man Lam Lam Foucks, S., Gonzales, M., AdelaideMerlande, J., Zonzon, J. et Alexandre, R., Regards sur l'histoire de la Caraïbe, Ibis Rouge.

- Durkheim, E. (1912). Les formes élémentaires de la vie religieuse. Paris : Alcan

- François, S. (2001). Les pirogues du Maroni, Enquête thématique, rapport de recherche. Association Aïmara pour le service régional de l'inventaire, oct-déc.

- Goreaud, J. (1931). « Mission Monteux-Richard 15 décembre 1930 - 15 septembre 1931 », manuscrit dactylographié.

- Guttman, A. (1979). From ritual to records. New York : Columbia University Press,

- Le Goff, P. (1996). Jeux, identités, pratiques. Le palet sur terre en basse Bretagne. Thèse de doctorat, sous la direction de J. M. Faure, Université de Provence,

- Mam Lam Fouck, S. (1999). La Guyane française au temps de l'esclavage, de l'or et de la francisation (18021946). Éditions Ibis Rouge.

- Maufrais R., Crunelle G. (1991). L'Appel de l'aventure, Editions Caribéennes. Paris : L'Harmattan (Réédition de 1949).

- Moomou, J. (2004). Le Monde des Marrons du Maroni en Guyane (1772-1860). La naissance d'un peuple: les Boni. Éditions Ibis rouge.

- Pegard, O. et Pruneau, J. (2001). La route du poisson : le sport au service du patrimoine. Ethnologie française, 31(1), 161-168

- Price, R. et S. (2003). Les Marrons. Vents d'ailleurs.

- Pruneau, J. (2003). Les joutes languedociennes. Ethnologie d'un « sport traditionnel ». Paris : L'Harmattan.

- Pruneau, J., Dumont, J. et Celimène, N. (2006). «Voiles traditionnelles aux Antilles françaises : « sportivisation » et « patrimonialisation ». Ethnologie française, 36(3), 519-530.

- Pfister, G. (1999). Les athlètes comme gérants du sport, spectateurs et arbitres. In J. M. Delaplace (dir.), L'Histoire du sport, l'histoire des sportifs (pp.311-324) Paris : L'Harmattan,

- Rapport de tournée dans l'Inini (Guyane française), période 1936-1942. Paris 1960.

\section{NOTES}

1. Le mot « marron » vient de l'espagnol « cimarrón »; il désignait les animaux domestiques qui, après s'être échappés, redevenaient sauvages. Concernant les esclaves, il est défini comme étant "le nègre qui s'est enfui de l'habitation de son maître et qui se cache dans les bois, les cavernes, les montagnes, pour échapper aux châtiments rigoureux dont on l'accable » (Larousse, 1991 : 1248)

2. Si l'orthographe de ce mot n'est pas clairement définie, il semble qu'il soit utilisé au singulier. Dans le langage des Bushinengué (bushinengué tongo) toutes les lettres sont prononcées.

3. Le terme de « Boni » était employé par les colonisateurs en référence au nom du deuxième chef guerrier du groupe qui s'est opposé à la domination espagnole. S'il est repris par certains auteurs ou par les autres populations de la Guyane, ce groupe Bushinengué ne se désigne pas ainsi. Il utilise plutôt le terme "Aluku », nom de leur premier leader, moins guerrier, tendant à euphémiser l'image de tribu dangereuse véhiculée par Boni Okilifu. D'ailleurs, en 1985 un Conseiller général Aluku, Antoine Abianso, a revendiqué cette appellation. De plus, en Guyane on parle de culture et d'art « Aluku » et non « Boni ».

4. Fleuve qui sépare la Guyane française du Surinam et qui est le cours principal du fleuve Maroni.

5. En 1712, pour échapper à l'impôt de capitation imposé au Surinam à titre de rançon par l'amiral français Jacques Cassard, les colons hollandais ont envoyé dans la forêt un bon nombre de leurs esclaves dans l'espoir de les récupérer par la suite.

6. Entretien du 6 mars 2005 réalisé à Cayenne par N. Agoudouman.

7. Le Maroni est le plus long fleuve de Guyane. Il prend naissance dans la région du Tumuc-Humac, sous le nom de Litani qu'il conserve jusqu'à sa confluence avec l'Inini. Il s'appelle ensuite Lawa puis Maroni après avoir reçu le Tapanahoni. En Guyane, la distinction entre Lawa et Maroni est rarement faite et il est fréquent d'entendre les gens situer la commune de Maripasoula sur le fleuve Maroni alors qu'en réalité, elle est sur le Lawa. 
8. Date à laquelle le conseil général a construit une route en terre battue qui relie Maripasoula à Boniville en passant par Papaïchton et Loca. Mais cette route n'est pas toujours praticable. C'est le cas lors de la saison des pluies où elle est boueuse, voire inondée sur certaines portions, notamment entre Loca et Boniville.

9. Moomou Jean, entretien du 31/12/04 à Boniville.

10. Farine grillée et granuleuse, de couleur jaune, obtenue à partir du manioc amer cuit jusqu'à complète déshydratation. Le couac est généralement mouillé avant consommation sauf dans les cas où il est mangé avec de la wassaï, du comu et du patawa et lors des repas en sauce (selon la préférence de chacun).

11. Quelqu'un qui soigne les malades par des plantes médicinales (tradipraticien).

12. Les Bushinengué sont des peuples polygames

13. Les feuilles utilisées peuvent provenir de différents palmiers : tachi, awaa bong, pina, kéé maka bong, maïpa bong.

14. Cérémonie qui a lieu environ huit jours après l'enterrement. Elle peut être comparée à la messe de huitaine. 15. Partie de pêche qui consiste à répartir dans une portion choisie du fleuve la sève d'une liane préalablement écrasée à l'aide d'un bâton. Le mélange de cette sève à l'eau endort les poissons de cette zone, les pêcheurs n'ayant plus qu'à les ramasser.

16. Cérémonie qui se déroule généralement un an après le décès de la personne et pendant laquelle le veuf ou la veuve sort du deuil. Lors de cette fête, des boissons notamment du rhum est versé à terre et de la nourriture est donnée aux morts.

17. L'Inini est aussi un affluent du Lawa.

18. Un placer est un gisement alluvionnaire riche en minéraux lourds ou précieux, comme l'or, la platine, le diamant.

19. Ibid.

20. Entretien du 17 août 2005 à Maripasoula.

21. La marchandise transportée à l'époque était liée aux trouvailles et aux déplacements des orpailleurs et des expéditeurs.

22. Entretien du 6 mars 2005.

\section{ANNEXES}

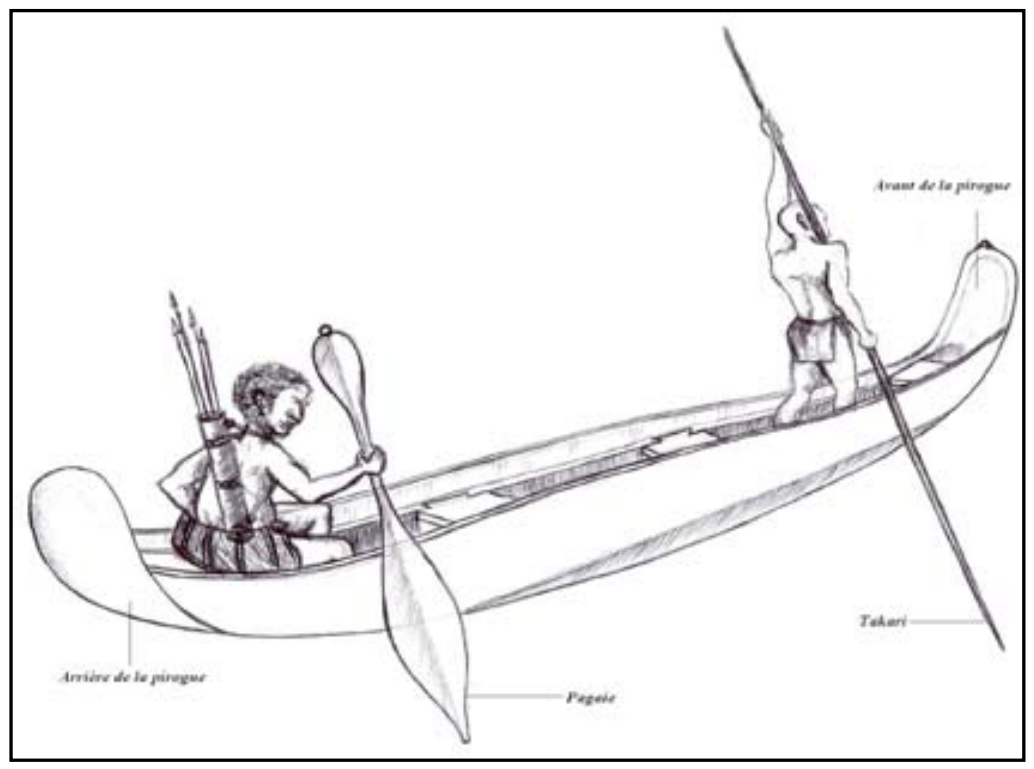

Figure 1 : une pirogue à pagaie avec en apparence une pagaie et un takari (dessin de P. AMAÏKON) 
Figure 2 : un takari ou kula (dessin de P. AMAÏKON)

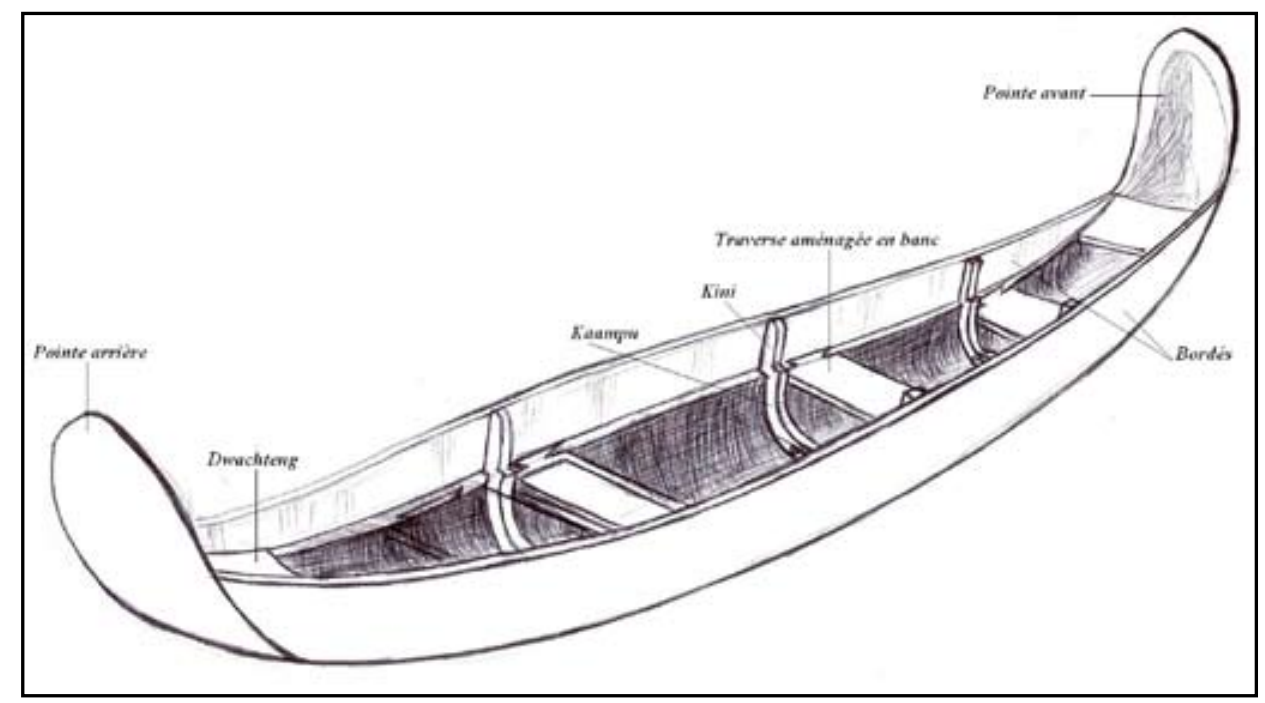

Figure 3 : une pirogue à pagaie terminée (dessin de P. AMAÏKON)

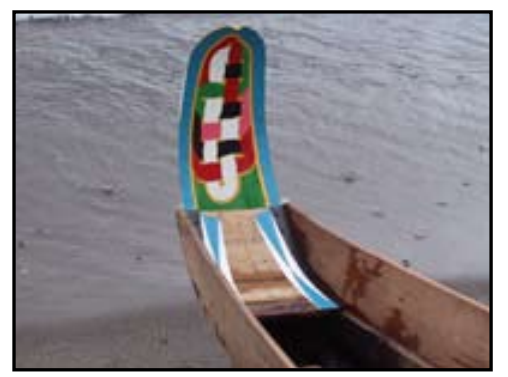

Figure 5 : La pointe avant d'une pirogue Djuka (photo de D. Millepied) 


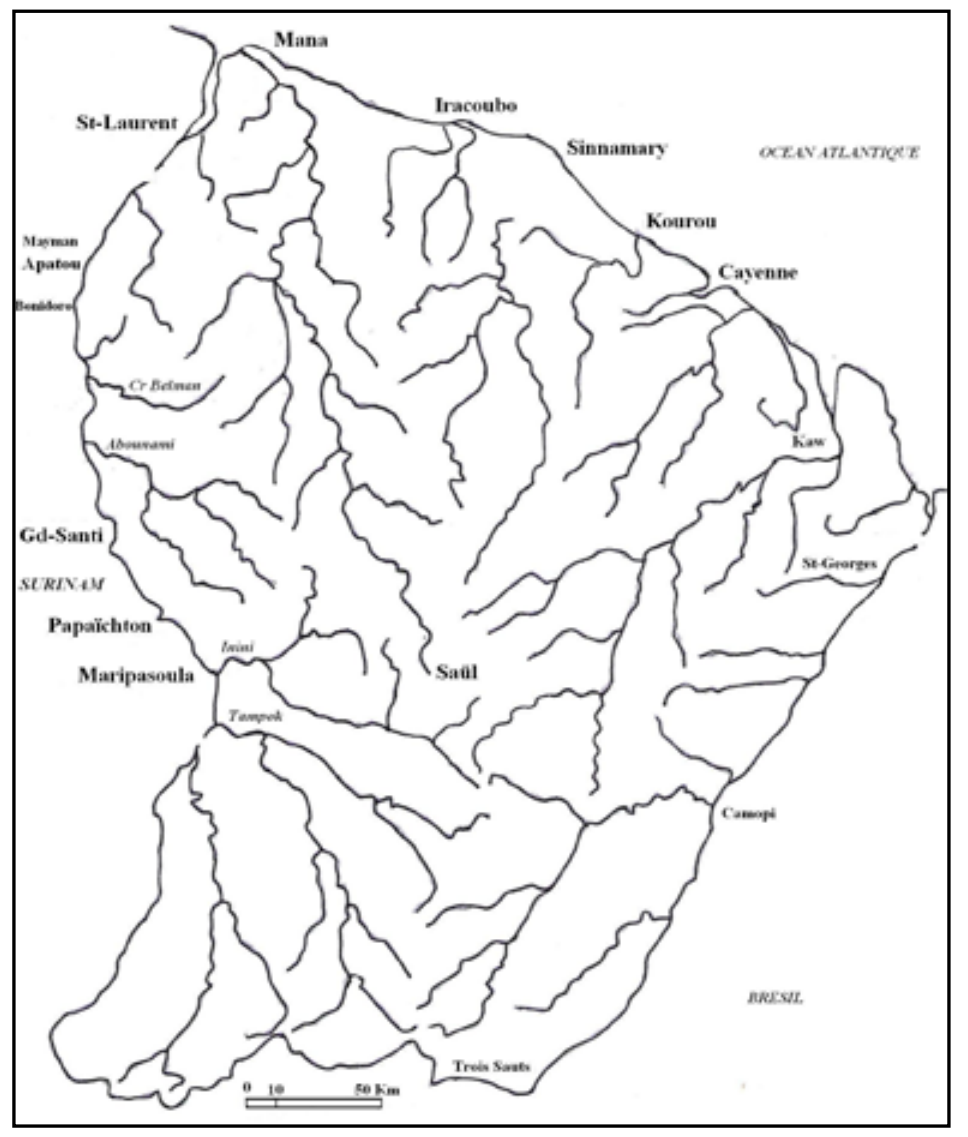

Figure 6 : Carte de Guyane 